\title{
Galactic Dynamics and Magnetic Field Amplification
}

\author{
HARALD LESCH \\ Max-Planck-Institut für Radioastronomie, Auf dem Hügel 69, W-5300 Bonn 1, FRG
}

November 24, 1992

\begin{abstract}
Stimulated by recent high frequency radio polarization measurements of M83 and M51, we consider the influence of non-axisymmetric features (bars, spiral arms, etc...) on galactic magnetic fields. The time scale for the field amplification due to the non-axisymmetric velocity field is related to the time scale of angular momentum transport in the disk by the non-axisymmetric features. Due to its dissipational character (cooling and angular momentum transport) the gas plays a major role for the excitation of non-axisymmetric instabilities. Since it is the gaseous component of the interstellar gas in which magnetic field amplification takes place we consider the interplay of gasdynamical processes triggered by gravitational instabilities and magnetic fields. A comparison with the time scale for dynamo action in a disk from numerical models for disk dynamos gives the result that field amplification by non-axisymmetric features is faster in galaxies like M83 (strong bar) and M51 (compagnion and very distinct spiral structure), than amplification by an axisymmetric dynamo. Furthermore, we propose that axisymmetric gravitational instabilities may provide the turbulent magnetic diffusivity $\eta_{T}$. Based on standard galaxy models we obtain a radially dependent diffusivity whose numerical value rises from $10^{25} \mathrm{~cm}^{2} \mathrm{~s}^{-1}$ to $10^{27} \mathrm{~cm}^{2} \mathrm{~s}^{-1}$, declining for large radii.
\end{abstract}

\section{Introduction}

Recently Neininger (1992) and Neininger et al. (1991) reported most sensitive $\lambda 2.8$ $\mathrm{cm}$ radio continuum measurements of M51 and M83, using the Effelsberg $100 \mathrm{~m}$ telescope. At this frequency Faraday rotation is negligible, thus these polarization measurements show the intrinsic large-scale structure of the magnetic field.

The magnetic field in M51 follows exactly the optical spiral arms. In particular, local deviations from the grand design spiral pattern are surprisingly well reproduced in the magnetic field morphology. It was concluded, that beside the action of a dynamo, the structure of the magnetic field may be explained as the response of the field to the particular dynamic pattern.

In M83, a barred spiral, similar features were observed; the magnetic field closely follows the the optical structure. The field changes its direction by $90^{\circ}$ at the ends of the bar and runs along the bar through the central region. Outside the bar it follows the optical arms. Again it was concluded, that gasdynamical processes which are related with the excitation of spiral structure or bars may be relevant for the observed magnetic field structure.

It is the purpose of this contribution to consider possible interactions of axisymmetric (clouds) and non-axisymmetric (spirals, bars, etc..) gravitational instabilities with galactic magnetic fields.

\section{Non-axisymmetric gravitational instabilities and spiral structure}

Spiral patterns are most prominent in galaxies which contain gas and are forming stars. In many cases they have an underlying "grand design", but the symmetry is almost always broken: fragmentary patterns and branching arms are very common. 
A complete theory should account in a unified way for the underlying symmetries, the lack of perfection, and the importance of gas.

The central twin problems of theories for spiral structure are the origin and persistence of the grand design. There is now little doubt that bars and compagnions are capable of driving grand-design spiral responses in gaseous and stellar disks (see Athanassoula 1984 for a review).

Attempts to establish a theory of long-lived spiral density waves began with Lin and Shu $(1964,1966)$ who showed that tightly wrapped spiral density waves were admissible free oscillations of a stellar disk. Toomre (1969) however, demonstrated that such waves require continous regeneration, because they propagate radially with a substantial group velocity. Recurrent transient spirals have already been proposed by Goldreich and Lynden-Bell (1965), Julian and Toomre (1966) and Toomre (1981). They find substantial, but limited, growth as leading spirals swing to trailing in a shearing disk. The decline in the strength of the patterns is caused by a steady rise in random motion (heating) amongst the disk stars brought about by the fluctuating potential perturbations of the spirals themselves.

The velocity dispersion in a stellar disk is conveniently expressed in terms of the parameter $Q$, which measures the ratio of radial component of velocity dispersion $v_{s}$ to the minimum required to suppress axisymmetric gravitational instabilities (Toomre 1964); viz.

$$
Q=\frac{v_{s} \kappa}{3.36 G \Sigma}
$$

here $\kappa$ is the local epicyclic frequency, and $\Sigma$ is the local surface density. A Q of 1 is sufficient for axisymmetric stability, but non-axisymmetric instabilities continue until $\mathrm{Q}$ has risen to somewhere between 2 and 2.5. The value of $Q$ has to be maintained under 2.5 if recurrent spiral activity is to be prolonged. This may be achieved in two different ways:

1. Dissipation of the gas layer. Collisions between gas clouds will prevent them from acquiring high random velocities.

2. Accretion. As material is added to the disk, the surface density will rise, which reduce the value of $Q$.

Both effects are intrinsic properties of non-axisymmetric instabilities:

Since the clouds crowd in the spiral arms they collide more often and since this kind of dissipation leads to a transfer of angular momentum, accretion will set in as a result of the excitation of spiral arms (see next section).

Sellwood and Carlberg (1984) (SC) presented computer simulations of a selfregulation mechanism for spiral instabilities in galactic disks. They allowed for cooling and accretion and argued that spirals are transient features in an evolving disk and that dissipation in the gas component is essential for the continued recurrence of spiral instabilities.

SC observed continuous spiral activity for as long as the models run. Each individual spiral pattern lasts for less than a rotation period $\tau \simeq \frac{2 \pi}{\Omega}$, but patterns succeed each other so rapidly that models always have spiral appearance. 
Thus, the role of gas is evident, only this component can cool fast enough to sustain the onset condition for non-axisymmetric instabilities. The $Q$ value of the stellar and gaseous component is only loosely connected. Thomasson et al. (1991) reported numerical simulations in which the stellar $Q$ stays constant, whereas the gaseous $Q$ varies considerably with radius and time.

\subsection{BARS}

Bars spontaneously form if, for self-gravitating masses, the criteria $T / W \geq 0.14$ and $Q<3$ are fulfilled (Ostriker and Peebles 1973). T is the rotational kinetic energy and $W$ is the potential energy. In other words, if most of the kinetic energy of the disk is in rotational rather than in random motion, the disk is strongly unstable to large-scale barlike modes.

The bar leads to a gas response which is faster than the rotation outside corotation CR (where the rotation frequency $\Omega$ is equal to the bar rotation frequency $\Omega_{p}$ ) and lags it inside CR, thus giving rise to torques which act on the gas to drive inwards inside CR and outwards outside CR. The inflow time scale for any non-axisymmetric distortion, which is equal to the time scale for a redistribution of angular momentum in the disk, is given by (Lynden-Bell and Kalnajs 1972; Larson 1984)

$$
\tau \simeq 2\left(\frac{M}{M_{d i s k}}\right)\left(\frac{\Sigma}{\delta \Sigma}\right)^{2} \frac{2 \pi}{\Omega} .
$$

$M \simeq v_{r o t}^{2} R / G$ is the total mass interior to radius $\mathrm{R} ; M_{\text {disk }} \simeq 2 \pi \Sigma$ is the disk mass interior to $\mathrm{R}$ and $v_{\text {rot }}=R \Omega$ is the rotation velocity. The non-axisymmetric disturbance of the gravitational potential produces a disturbance in the surface density $\delta \Sigma$.

Eq. (2) shows that if most of the mass is in the disc, $\left(M / M_{d i s k} \sim 1\right)$ and if a nonaxisymmetric disturbance of large amplitude and wavelength is present $\Sigma / \delta \Sigma \sim 1$, the time scale for redistribution of angular momentum is of the same order as the orbital period, in accordance with the results of SC.

The condition $\delta \Sigma \sim \Sigma$ shows again the importance of the gaseous component. It has been shown by Lubow et al. (1986) that for a gas to star ratio of only $15 \%$, the gas contributes seven times more to self-gravity than the stars. The main reason for the importance of gas gravity is that a stellar disturbance is a fractionally small perturbation on an otherwise axisymmetric stellar disc, while the gas is fully participating. In that case only a small fraction of the stellar density generates a bar or a spiral wave, while much of the gas density generates a spiral field or a bar. So that the assumption $\delta \Sigma \sim \Sigma$ is easily fulfilled for the gas, for even weak disturbances in the stars.

\section{Axisymmetric gravitational instabilities and star formation}

A galactic disk is unstable to axisymmetric gravitational instabilities (GI) if $Q<1$ (Toomre 1964). The GI leads to a growth of small density perturbations and causes a rotating disk to fragment into clumps. The angular momentum transport is then 
provided by the shear and the viscosity produced this instability. The viscosity is related to the clouds that form in a locally gravitational unstable disk. The forming fragments have a scale and separation of order $\lambda_{c}$ given by

$$
\lambda_{c}=\frac{2 \pi^{2} G \Sigma}{\kappa^{2}}
$$

and their velocity

$$
v_{c}=\frac{\pi G \Sigma}{\kappa} .
$$

The disk is marginally stable for $v_{s}=v_{c}$ (we note that $Q=\frac{v_{s}}{v_{c}}$ ).

We assume that the disc is kept on the border of GI, creating a cloudy medium with both cloud sizes and separation of order $\lambda_{c}$. These clouds should provide the angular momentum transport by a two-dimensional random walk with a step length $\lambda_{c}$ and velocity $v_{c}$, i.e. the viscosity is (Lin and Pringle 1987)

$$
\nu \sim \lambda_{c} v_{c} \sim \lambda_{c}^{2} \Omega(R)
$$

From the onset condition $Q<1$, Kennicut (1989) defined a critical gas surface mass density $\Sigma_{c}$. He found that in disk galaxies the observed surface mass density is larger than $\Sigma_{c}$. He concluded that the star forming threshold appears to be associated with the onset of large-scale GI in the gas disk.

Thus, GI may serve as a large-scale source for turbulence in the interstellar medium; we will discuss this assumption and its application to dynamo action in galaxies in the last section.

Here we proceed in relating the same dynamical processes which may be responsible for the disk structure to the magnetic field structure.

\section{Magnetic field amplification and spiral arms}

Any density disturbance in a magnetized disk, which moves with high velocity, intensifies the component of magnetic field which is tangential to the disturbance. From the dynamical considerations it is clear, that any non-axisymmetric perturbation leads to angular momentum transfer and thus to a radial inflow.

Thus, it seems reasonble to approximate the dynamics of a disk galaxy which contains spiral arms or bars or is interacting, by a two-dimensional velocity field $\left(v_{r},, v_{r o t}, 0\right)$. We use the induction equation, with dissipation neglected (Ruzmaikin et al. 1988, Sect. VII),

$$
\frac{\partial \mathbf{B}}{\partial t}=\nabla \times(\mathbf{V} \times \mathbf{B}) .
$$

For the azimuthal field component $B_{\varphi}$ one gets

$$
\frac{\partial B_{\varphi}}{\partial t}=-\frac{\partial}{\partial r}\left(v_{r} B_{\varphi}\right)+B_{r} r \frac{d \Omega}{d r}
$$

with $B_{r} \sim$ const. 


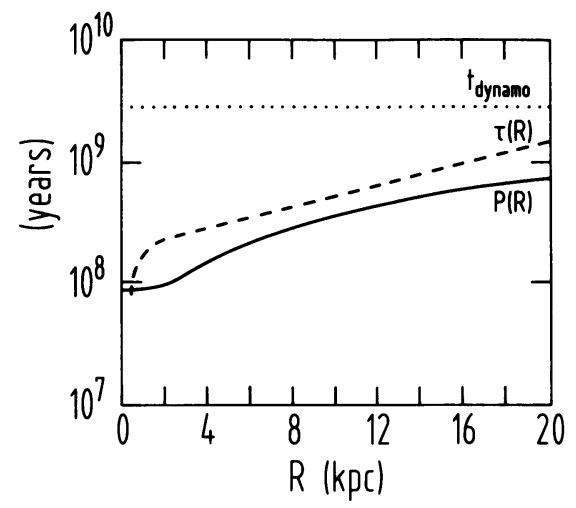

Fig. 1. The dynamo time scale $t_{\text {dynamo }}$ (Eq. (9)) (dotted line), the time scale $\tau$ (Eq. (2) and (8)) for exponential growth of the toroidal field due to amplification by non-axisymmetric perturbations (broken line) and the rotation period $P(R)$ (solid line) in a "standard" galactic disc.

The solution to this equation grows exponentially as

$$
B_{\varphi}=\left[B_{\varphi 0}\left(r_{0}\right)+\tau B_{r} r \frac{d \Omega}{d r}\right] \exp \left(\frac{t}{\tau}\right)-\tau B_{r} r \frac{d \Omega}{d r}
$$

where the amplification time is

$$
\tau=-\left(\frac{\partial v_{r}}{\partial r}\right)^{-1}
$$

Eq. (8) describes the same time scale as Eq. (2). Thus, we compare this time scale with the time scale an $\alpha \omega$-dynamo needs to amplify a magnetic field. Here we use the numerical simulations of Camenzind and Lesch (1992) which describe the time evolution of axisymmetric magnetic fields in galactic disks. It is known, that axisymmetric fields have the highest growth rate, i.e. the smallest time scale. Its typical growth time is (Camenzind and Lesch 1992)

$$
t_{\text {dynamo }} \simeq \frac{R_{b u l g e}^{2}}{\eta_{T}\left(R_{b u l g e}\right)} \simeq 10^{9} \text { yrs }\left[\frac{R_{b u l g e}}{3 k p c}\right]^{2}\left[\frac{\eta_{T}}{3 \cdot 10^{26} \mathrm{~cm}^{2} s^{-1}}\right]^{-1}
$$

$R_{b u l g e}$ is the bulge radius, where the differential rotation of the disk evolves into rigid rotation of the central bulge. There, the gradient of differential rotation is highest and thus, it acts as the source for the dynamo action. The amplified flux diffuses radially outwards. As the source of the turbulent diffusivity we take the 


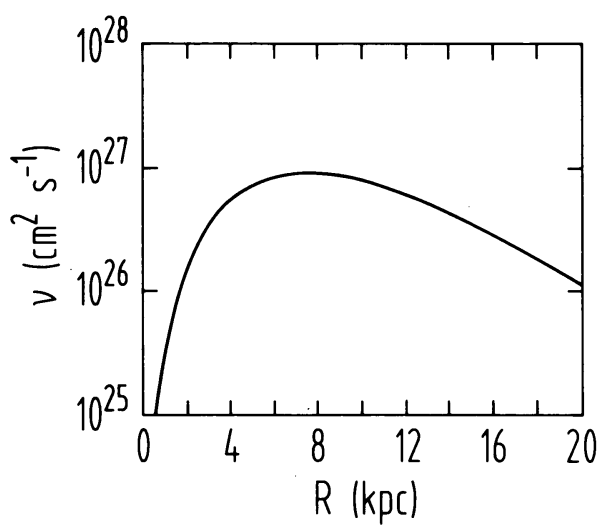

Fig. 2. The radial profile of the turbulent diffusivity $\eta_{T}$. Here it is assumed that axisymmetric gravitational instabilities provide the viscosity $\nu$ (Eq. 5) in the disk and that the viscosity is equivalent to a turbulent diffusivity.

axisymmetric gravitational instability. As mentioned above one can define a viscosity driven by the GI. Since disk galaxies are locally unstable against GI (Kennicut 1989) this assumption seems to be reasonable.

In Fig. 1 we show the time scales $\tau$ (Eq. (2) and (8)), $t_{\text {dynamo }}$ (Eq. (9)). and the rotation period $P(R)=2 \pi / \Omega(R)$ for a rotation curve of the form ( $\Omega_{0}$ is the rotation frequency of the rigidly rotating central region)

$$
\Omega(R)=\frac{\Omega_{0}}{\left[1+\left(\frac{R}{R_{b u l g e}}\right)^{1 / 3}\right]^{3}},
$$

which presents a general rotation curve of a disk galaxy. Furthermore we assume an exponential for $\Sigma$. Obviously the amplifcation by a non-axisymmetric perturbation is much faster than an axisymmetric dynamo.

In Fig. 2 we show the turbulent diffusivity as a function of radius, for a galaxy with an exponential disk and $\Omega(R)$ given by Eq. (10). The numbers we obtain with our assumption that GI are responsible for $\eta_{T}$ are very close to the values of $\eta_{T}$ deduced from observations of the turbulence in the interstellar medium (Ruzmaikin et al. 1988).

\section{References}

Athanassoula, E.: 1984, Phys. Rep. 114, 321

Camenzind, M., Lesch, H.: 1992, $A A$ (in press) 
Goldreich, P., Lynden-Bell, D.: 1965, MNRAS 130, 125

Julian, W.H., Toomre, A.: ApJ 146, 810

Kennicutt, R.C.:1989, ApJ 344, 685

Larson, R.B.: 1984, MNRAS 206, 197

Lin, C.C., Shu, F.H.: 1964, ApJ 140, 646

Lin, C.C., Shu, F.H.: 1966, Proc. Nat. Acad. Sci. 55, 229

Lin, D.N.C., Pringle, J.E.: 1987, MNRAS 225, 607

Lubow, S.H., Balbus, S.A., Cowie, L.L.: 1986, ApJ 309, 496

Lynden-Bell, D., Kalnajs, A.J.: 1972,MNRAS 157, 1

Neininger, N.: 1992,AA 263, 30

Neininger, N., Klein, U., Beck, R., Wielebinski, R.: 1991, Nature 352, 781

Ostriker, J.P, Peebles, P.J.E.: 1973, ApJ 186, 467

Ruzmaikin, A.A., Shukurov, A.M., Sokoloff, D.D.: 1988, Magnetic Fields of Galaxies, Reidel, Dordrecht

Sellwood, J.A., Carlberg, R.G.: 1984, ApJ 282, 61

Thomasson, M., Donner, K.J., Elmegreen, B.G.: 1991, AA 250, 316

Toomre, A.: 1964, ApJ 139, 1217 